\title{
Age-related changes in spatial discrimination learning performance in Lewis rats
}

\author{
ARJAN BLOKLAND, WIEL HONIG, and WIJNAND RAAIJMAKERS \\ University of Limburg, Maastricht, The Netherlands
}

\begin{abstract}
Previous studies have shown that spatial discrimination learning is preserved in old Lewis rats in a spatial cone-field task. In the present study we evaluated the spatial discrimination performance of Lewis rats of different ages in the spatial Morris task. Compared with young and middle-aged rats, old rats showed a performance deficit in the Morris task. The use of spatial cues was assessed by using a novel procedure in which the platform was moved to different rooms. In addition, the spatial bias was assessed in a probe trial procedure. It was concluded that the poor performance of the old rats in the Morris task was a result of their not using spatial cues to solve the task. The results of the present study suggest that age differences in performance in spatial learning are task dependent for Lewis rats. This in turn suggests that age-related deficits in spatial discrimination performance may not always reflect deficits in spatial information processing.
\end{abstract}

In the last decade, much attention has been given to animal models of the decline in learning and memory performance that is associated with aging. These animal models are valuable tools in the study of mechanisms involved in learning and memory, and they make it possible to assess treatments for the disturbances in learning and memory processes that accompany aging in humans. A decline in learning and memory performance in aged rats has been found in short-term-memory tasks (e.g., Dunnett, Evenden, \& Iversen, 1988; Wallace, Krauter, \& Campbell, 1980) and in spatial learning tasks (e.g., Barnes, Nadel, \& Honig, 1980; Rapp, Rosenberg, \& Gallagher, 1987; van der Staay, Krechting, Blokland, \& Raaijmakers, 1990). Consequently, tasks that measure either short-term-memory processes or spatial discrimination learning are assumed to be valid models to test the age-related decline in learning and memory (Gallagher \& Pelleymounter, 1988; Olton, 1988; Sarter, 1987).

Age-related performance deficits have been found in different tasks measuring spatial learning and memory in the rat (see Rapp et al., 1987). However, when comparing the performance of young and old subjects in learning and memory tasks, one should control for agerelated differences in factors that could interfere with learning and memory performance-for example, speed of responding, response strategies, and motivation (see Ingram, 1985; Rigter, 1971; Sarter, 1987; van der Staay, Krechting, et al., 1990), as these factors could influence the validity of the model used. If, for example, rats of different ages use different problem-solving strategies,

Preliminary results of a part of this study have been discussed in Raaijmakers, Blokland, and van der Staay (1993). Address correspondence to A. Blokland, Institute for Neurobiology, Department of Gerontopharmacology, Troponwerke, Berliner Strasse 156, D-51063 Cologne, Germany. the performance of young and old rats cannot be directly compared.

The Morris spatial navigation task has been used to reveal age-related performance deficits (e.g., Gage, Dunnett, \& Björklund, 1984) that are assumed to be caused by an impairment in spatial information processing in old rats (Rapp et al., 1987). This suggests that the Morris task can be used to study the age-related decline in learning and memory. Studies that report that rats show impairments in the Morris task after hippocampal lesions support the notion that rats use spatial information in this task (Morris, Garrud, Rawlins, \& O'Keefe, 1982; Morris, Schenk, Tweedie, \& Jarrard, 1990; Whishaw, 1987). Furthermore, it has been found that cholinergic antagonists impair spatial navigation (e.g., Whishaw, 1985). This indicates that the Morris task is sensitive to cholinergic dysfunction, which is, according to Lindner and Schallert (1988), a prerequisite for an animal model of aging.

However, the occurrence of an age-related performance deficit in the Morris task is not consistent over rat strains and experiments. For example, Lindner and Schallert (1988) reported no age-related deficits in spatial learning in the Morris task with Long-Evans rats, although an age-related decline in performance was found with Fischer rats. This preserved spatial discrimination learning performance of old Long-Evans rats stands in contrast to the results of most other studies reporting performance deficits in the same strain (Aitken \& Meaney, 1989; Pelleymounter, Smith, \& Gallagher, 1987; Rapp et al., 1987). Apart from the study of Rapp et al., other studies have reported that old Long-Evans rats eventually do not perform as well as young rats (Aitken \& Meaney, 1989; Pelleymounter et al., 1987). Old Sprague-Dawley and Fischer rats do not seem to reach a level of performance similar to that of young 
rats (Gage et al., 1984; Lindner \& Schallert, 1988). Although there were procedural differences between these experiments, the findings suggest that there may be strain differences in spatial discrimination learning performance, which may have consequences for the validity of this model.

We have reported strain differences in spatial discrimination learning performance in a complex, spatial cone-field task. In this task, food-deprived rats are started from four, randomly chosen, different positions and have to find food hidden in the top of a subset of 16 cones, which are kept in a fixed position during training (see van der Staay, Blokland, \& Raaijmakers, 1990; van der Staay, Krechting, et al., 1990). Although a consistent age-related decline in spatial discrimination learning was found in Brown-Norway rats (van der Staay, Krechting, et al., 1990), we found that old (18-, 24-, and 30-month-old) Lewis rats had a better spatial discrimination performance in a spatial cone-field task than young (4- and 12-month-old) rats (van der Staay, Blokland, \& Raaijmakers, 1990). This stands in contrast to the notion that spatial discrimination learning is impaired in old rats. We assumed that young Lewis rats adopted the habit of inspecting nonreinforced places in the cone-field task, which resulted in an age-related "increase" in spatial discrimination performance. In a simpler cone-field task, we found no age-related spatial discrimination deficit in Lewis rats and found that both young and old rats used an allocentric orientation strategy in this task (Blokland, Raaijmakers, Honig, \& van der Staay, 1992). Thus, old Lewis rats can use spatial cues in the cone-field task.

In the present study, we evaluated the spatial discrimination performance of Lewis rats of different ages in the Morris spatial navigation task. The cone-field task can be regarded as being more complex than the Morris task. Rats have to search for food in four different locations in the cone-field task, whereas only one position has to be remembered in the Morris task. Furthermore, in the spatial cone-field task, young and old rats need extensive training (50 and 60 trials, respectively) to reach an asymptotic performance that is not errorless (about seven visits to collect pellets from four baited cones). Apart from the difference in task complexity, the Morris swim task has a more aversive character than the cone-field task. This could prevent young Lewis rats from developing the habit of exploring the water tank, which could interfere with spatial discrimination performance. Because old Lewis rats performed well in the more complex cone-field task, we hypothesized that old Lewis rats would perform as well as young rats in the Morris task.

We first evaluated age differences in the performance of the Morris task by testing Lewis rats of three different ages. After the rats had reached an asymptotic performance, we introduced a novel procedure to evaluate whether the rats used spatial cues to find the platform position. The water tank was moved to different rooms with novel cues. If the rats used spatial cues in one room, the escape latency would be higher than the asymptotic performance during the initial trials of testing in a new environment. However, if the rats did not use spatial cues in one room, the escape latency during the initial trials would be similar to that of the asymptotic performance. In a second experiment, we tested young and old rats in the Morris task and used a probe trial procedure to evaluate spatial bias.

\section{EXPERIMENT 1A}

\section{Method}

Animals and Materials. Male Lewis rats of three different ages were used: young ( 5 months old, $n=8$ ), middle aged ( 13 months old, $n=8$ ), and old ( 25 months old, range $23-27, n=7$ ). All rats were housed individually in standard Makrolon cages on sawdust bedding in an air-conditioned room (about $20^{\circ} \mathrm{C}$ ). They were kept under a 12:12-h light:dark cycle (lights on from 9:00 to 21:00). All the old rats were healthy and were regularly checked for their SPF status during testing. The old rats had no cataracts and showed no severe motor impairments - that is, no paralysis of the hind legs.

We observed that the death rate of the Lewis rats, which were kept in supply for experiments on aging in our animal house, started to increase from the age of 20 months, reaching a peak at about 28 months.

Behavioral procedure. The rats were trained on the Morris spatial navigation task (Morris, 1981) in a black water tank with a diameter of $1.22 \mathrm{~m}$. The rats were started from four different, randomly chosen positions ( $\mathrm{N}, \mathrm{E}, \mathrm{S}$, and $\mathrm{W})$ and were trained to find an invisible platform (diameter of $11 \mathrm{~cm}$ ) that was at a fixed position in the water tank, $1 \mathrm{~cm}$ below the surface of the water. The temperature of the water was $20^{\circ}-22^{\circ} \mathrm{C}$. A trial lasted until a rat had found the platform or until $60 \mathrm{sec}$ had elapsed. If a rat did not find the platform within $60 \mathrm{sec}$, it was placed on the platform for $3 \mathrm{sec}$ and then removed from the water tank. The rats were given 4 trials on the first day, and 8 trials per day thereafter on consecutive days. The young and middle-aged rats received a total of 28 trials, whereas the old rats received a total of 44 trials.

Statistical analysis. Differences in escape latency were analyzed per trial block of four trials on the first seven trial blocks. The performance of the rats during training was analyzed with a two-factor (age and trial blocks) analysis of variance (ANOVA) with repeated measures over trial blocks. In addition, differences in learning curves were analyzed by comparing the individual orthogonal trend components calculated over the first three trial blocks (Winer, 1971), which correspond to the first phase of acquisition. A Duncan-Waller post hoc analysis was performed on differences in overall performance (general mean) and on individual orthogonal trend components in order to specify the agerelated changes in more detail.

\section{Results}

The latency to find the platform increased with age on Trial Blocks $1-7(F \mathrm{~s}>3.78, p<.05$; see Figure 1$)$. The overall decrease in escape latency during the first phase of acquisition (Trial Blocks 1-3) was generally characterized by a linear trend $[F(1,20)=51.15, p<.01]$, which explained $95 \%$ of the variation. The age groups differed in the linear decrease in escape latency over the first three trial blocks [age $\times$ block interaction: $F(2,20)=$ $9.69, p<.01]$. Post hoc analysis revealed that the linear decrease in escape latency was similar in the young and middle-aged rats, but slower in the old rats. 


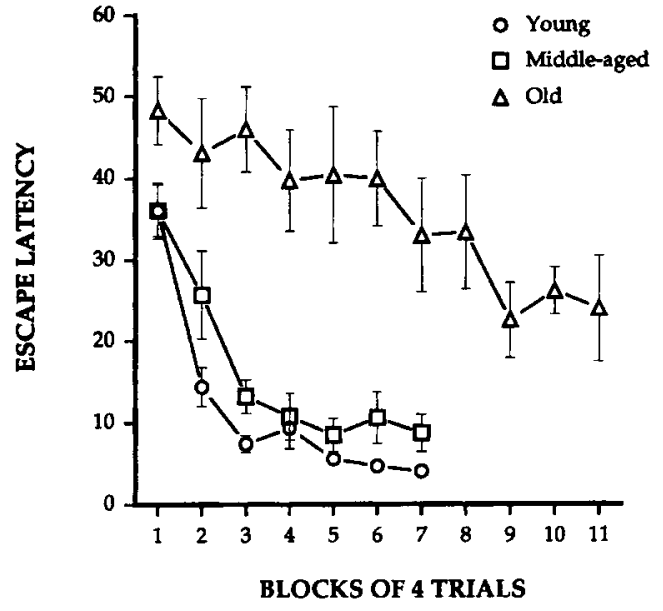

Figure 1: Mean escape latency per block of four trials (in seconds $\pm S E M$ ) of Lewis rats of different ages during acquisition of the Morris swim task with one platform position and four start positions (modified from Raaijmakers, Blokland, \& van der Staay, 1993).

Analysis of overall performance over the first seven trial blocks revealed that the three age groups had different performances [general mean: $F(2,20)=33.79$, $p<.01]$. Post hoc analysis showed that the performance of the old rats was worse than that of the young and middle-aged rats; the performance of the young and middle-aged rats was similar.

\section{EXPERIMENT 1B}

\section{Method}

Animals. The rats that were used in Experiment 1A were used.

Behavioral procedure. After the rats had reached an asymptotic level of performance in the standard Morris task (see Experiment $1 \mathrm{~A}$ ), the water tank was moved to another room. This room was not similar to the previous experimental room with respect to spatial cues. The rats were again trained on the same procedure as in Experiment 1:2 days for the old rats and 3 days for the young and middle-aged rats after the first acquisition training. The rats were given four trials on Day 1 and eight trials on Day 2. After 1 day, the water tank was again moved to another room. This room was somewhat similar to the second room, but instead of fluorescent strip lights on the ceiling, two spotlights $(60 \mathrm{~W})$ on the wall (spaced $2 \mathrm{~m}$ ) illuminated the room and provided distinct spatial cues. The rats were then trained for 2 days, as they were for the second acquisition training.

Statistical analysis. The escape latencies were analyzed in a three-factor (age, blocks, and acquisition) ANOVA. Repeated measures over acquisition were performed separately over the first and second acquisition training periods and over the second and third acquisition training periods. This was done to evaluate whether the mean escape latency of the rats decreased on successive acquisition training periods, which would imply that the rats had learned the procedural aspects of the task. A Duncan-Waller post hoc analysis evaluated the age-related differences in more detail. In order to evaluate whether the performance of the rats was affected by changing the environment, the following two difference scores were calculated: ([escape latency Block 1, Acquisition 2] - [mean escape latency last three trial blocks, Acquisition 1], and [escape latency Block 1, Acquistion 3] - [mean escape latency last three trial blocks, Acquisition 1]). The hypoth- esis that the difference score deviated from zero was tested by using $t$ statistics. Because of the multiple comparisons, the likelihood of making a Type I error increased. Therefore, the critical $t$ value was adjusted according to Dunn's multiple comparison test (Dunn, 1961).

\section{Results}

The mean escape latency decreased during the first three blocks of the second acquisition training period compared with that of the first three blocks of the first training period [acquisition effect: $F(1,20)=34.20$, $p<.01$; see Figures $2 \mathrm{~A}$ and $2 \mathrm{~B}$ ], indicating that the rats had learned the procedural aspects of the task. Although the old rats performed more poorly than the young and middle-aged rats [general mean: $F(2,20)=$ $22.80, p<.01]$, there was no age difference in the decrease in escape latency from the first to the second acquisition training periods [age $X$ acquisition interaction: $F(2,20)=2.34$, n.s.]. There was no decrease in escape latency from the second to the third acquisition training periods $[F(1,20)=1.33$, n.s.; see Figures $2 \mathrm{~B}$ and $2 \mathrm{C}]$. In the third acquisition training period, the old rats still had an impaired performance compared with that of the young and middle-aged rats [general mean: $F(2,20)=$ $6.40, p<.01]$.

Analysis of the difference scores revealed that the escape latency of the young rats increased during the first trial block of the second and third acquisition training periods $[t(7)=4.85, p<.01$, and $t(7)=5.66, p<.01$, respectively]. The performance of the middle-aged and old rats during the first block of the second and third acquisition training periods did not differ from the asymptotic performance $(t \mathrm{~s}<2.58$, n.s.). Although the performance of the middle-aged rats during the first trial blocks did not differ statistically from the asymp-

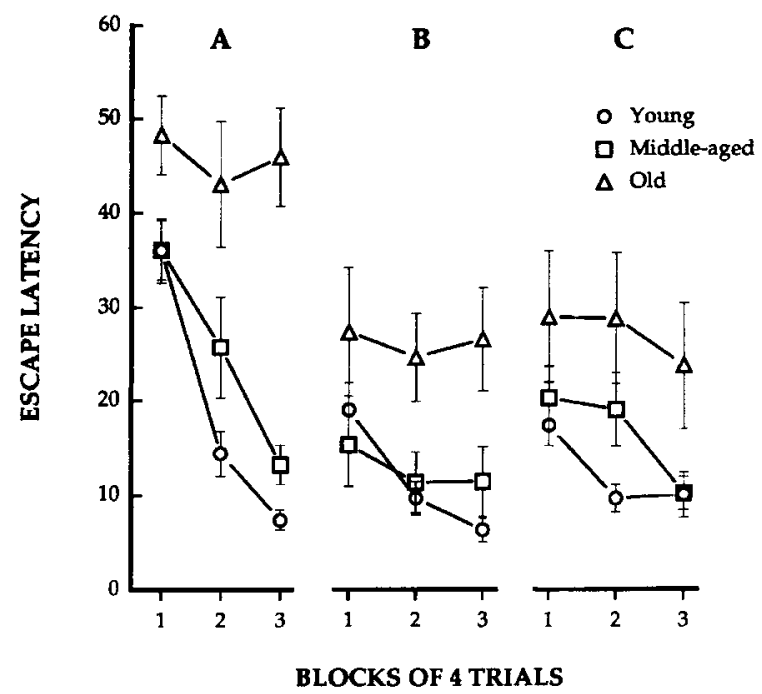

Figure 2. Mean escape latency per block of four trials (in seconds $\pm S E M$ ) of Lewis rats of different ages during the first three trial blocks of the first $(A)$, second $(B)$, and third $(C)$ acquisition training period of the Morris task with one platform position and four start positions. 
totic performance, there was an increase in the mean escape latency that was not found with the old rats.

\section{EXPERIMENT 2}

\section{Method}

Animals and Materials. Male Lewis rats of two different ages were used: young ( 5 months old, $n=8$ ) and old ( 25 months old, $n=8$ ). Housing conditions and selection criteria were similar to those described in Experiment $1 \mathrm{~A}$.

Behavioral procedure. The rats were trained on the Morris spatial navigation task, as described in Experiment $1 \mathrm{~A}$. However, the young and old rats were both given 28 trials. After the last trial, the rats were subjected to a probe trial lasting $60 \mathrm{sec}$. During the probe trials, the platform was removed from the water tank and the behavior of the rats was recorded with a video camera and then analyzed. The time spent per quadrant (NE, SE, SW, and NW) was recorded manually with an MS-DOS computer. The start position of the rats was randomly distributed over the four possible positions within both groups.

Statistical analysis. The performance of the rats during acquisition training was analyzed as described in Experiment $1 \mathrm{~A}$. For probe trials, the time spent in each quadrant was measured. Group differences in the time spent in the goal quadrant (NE) were analyzed with $t$ statistics. In addition, $t$ statistics were used to analyze whether the time spent in the goal quadrant differed from the chance level $(15 \mathrm{sec})$.

\section{Results}

The escape latencies of all the rats decreased in the course of training $[F(6,84)=13.26, p<.01$; see Figure 3]. As expected, the old Lewis rats performed more poorly than the young rats during the acquisition of the Morris task [general mean: $F(1,14)=21.05, p<.01$ ], and the escape latencies of the young rats decreased faster than those of the old rats [blocks $\times$ age interaction: $F(6,84)=6.15, p<.01]$. During the probe trial, the young rats spent more time in the training quadrant than the old rats did $[t(14)=3.89, p<.01$; see Fig-

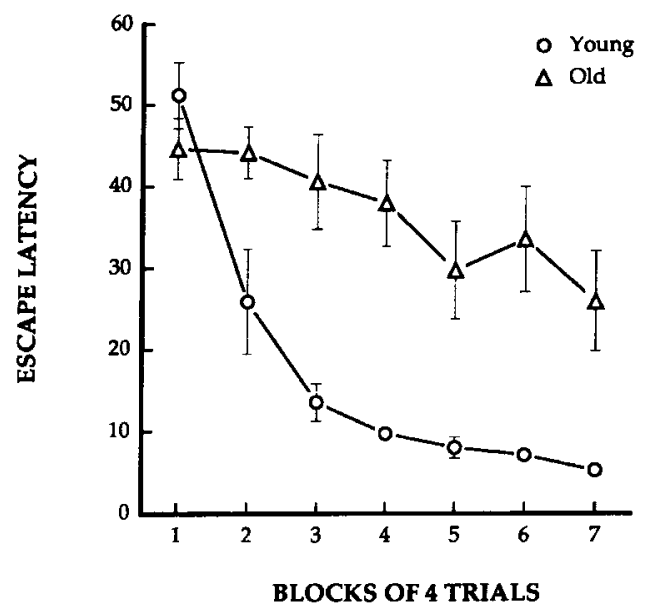

Figure 3. Mean escape latency per block of four trials (in seconds $\pm S E M$ ) of young and old Lewis rats during acquisition of the Morris swim task with one platform position and four start positions.

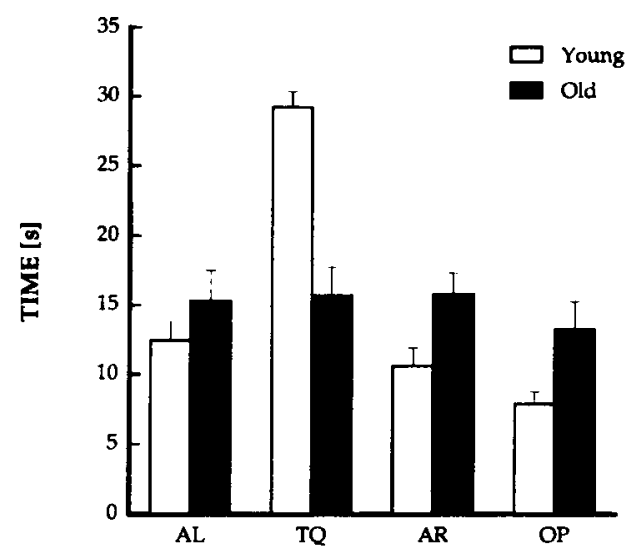

Figure 4. Time (+SEM) spent in the four different quadrants of the water tank (AL, adjacent left; $T Q$, training; $A R$, adjacent right; OP, opposite) during the probe trial for young and old Lewis rats in the Morris task.

ure 4]. The time that the old rats spent in the training quadrant did not differ from the chance level $[t(7)=$ 0.21 , n.s.], whereas the young rats spent more than $15 \mathrm{sec}$ in the training quadrant $[t(7)=12.45, p<.01]$. These data indicated that the young rats showed a spatial bias toward the previous platform position, but the old rats did not.

\section{DISCUSSION}

In the first experiment, the old rats were slower in learning a spatial navigation task and did not reach a level of performance similar to that of the young and middle-aged rats. These findings corroborate the impairment that is generally found in spatial discrimination performance in old rats (Barnes et al., 1980; Rapp et al., 1987; van der Staay, Krechting, et al., 1990). However, the impairment in spatial discrimination learning of the old Lewis rats in the Morris task contrasts with our previous finding that old Lewis rats reach a fairly high level of spatial discrimination performance in a more complex spatial cone-field task (van der Staay, Blokland, \& Raaijmakers, 1990) and perform as well as young rats in a simpler cone-field task (Blokland et al., 1992). We therefore assume that, at least for Lewis rats, the two spatial tasks measure different aspects of behavior. Moreover, young Lewis rats develop a habit of reinspecting nonrewarded places in the cone-field task (van der Staay, Blokland, \& Raaijmakers, 1990), and old rats do not use spatial cues in the Morris task (see below). Apparently, the age-related differences in spatial discrimination performance in Lewis rats, as measured in the cone-field task and the Morris task, may not reflect age-related changes in the processing of spatial information.

A possible explanation for this finding could be the use of different operationalizations of spatial discrimination performance that cannot be generalized over tests (see van Luijtelaar, van der Staay, \& Kerbusch, 
1989). In the present study, place navigation in the Morris task was operationalized as the time taken to find the platform (swimming time is highly correlated with other measures of performance in the Morris task; Gage et al., 1984), whereas place learning in the conefield has been operationalized as visits to baited cones in relation to visits to nonbaited cones (van der Staay, Blokland, \& Raaijmakers, 1990; van der Staay, Krechting, et al., 1990). Another important difference between the Morris task and the cone-field task is that the Morris task is aversively motivated (escaping from water), whereas the cone-field task is food motivated. In another study we did not find a correlation between spatial discrimination performance in the Morris and conefield tasks in young and old rats (Blokland \& Raaijmakers, 1993), which supports our notion that performance in both spatial tasks reflects different aspects of behavior in young and old Lewis rats. A lack of correlation between two spatial tasks (Morris task and circular platform task) in old rats has also been reported in another study with Sprague-Dawley rats (Markowska et al., 1989).

In Experiment $1 \mathrm{~B}$ we changed the environmental cues to evaluate whether the rats used an allocentric orientation strategy - that is, whether they used spatial cues. This experiment revealed that the escape latency of the old rats during Block 1 of the second and third acquisition training periods did not deviate from the asymptotic performance of the first acquisition training period. Thus, changing the environment (i.e., changing spatial cues) did not affect the performance of the old rats, which suggests that old rats do not use spatial cues in the Morris task. In contrast to the old rats, the escape latency of the young rats during Block 1 of the first and second acquisition training periods increased compared with that of the asymptotic performance. Apparently, the performance of the young rats was affected by changing the test environment, which indicates that they had used spatial cues to acquire the task.

In an additional experiment with young and old Lewis rats, we again found an age-related deficit in spatial discrimination performance in the Morris task. Using a more conventional procedure to evaluate the spatial bias of rats in the Morris task (i.e., the probe trial), we found that the young rats showed a spatial bias toward the training quadrant. In contrast, the old rats did not show a spatial bias and spent a comparable amount of time in the four different quadrants of the swimming pool. These results support the findings of the second experiment and suggest that old Lewis rats do not use spatial cues to optimize their search behavior in the Morris task.

Qualitative observations suggested that the old rats developed a strategy of making circular excursions from the wall of the water tank. This strategy increases the likelihood of "finding" the platform. In the course of training, the tendency to "hug the wall" decreased in the old rats, as they tended to swim in circles without returning to the wall very often. This change in behav- ior may explain the decrease in escape latency observed for the old rats during training.

In studies examining the age-related changes in rats' performance in the Morris task, spatial bias has been evaluated in probe trials. In probe trials, the platform is either removed (e.g., Pelleymounter et al., 1987) or moved to another position in the water tank (e.g., Gage et al., 1984). The spatial bias of the rats can be assessed by two measures: time spent in quadrant and annulus crossings (i.e., number of crossings of the previous platform position). Because old rats are more rigid in their behavior (Goodrick, 1975; but see Stephens, Weidmann, Quartermain, \& Sarter, 1985), they would be expected to spend more time in the training quadrant than young rats. Thus, an age-related decrease in the time spent in the training quadrant could be a clear indication that old rats have no spatial bias.

However, aged rats have been found to be more sensitive to proactive interference than young rats (Dunnett, Martel, \& Iversen, 1990). Furthermore, it has been suggested that old rats react more flexibly to withdrawal of reinforcement in a learning task (Sarter \& Markowitsch, 1983). An increased sensitivity to proactive interference and response flexibility in old rats could have affected the search behavior of the young and old rats differently in the probe trials, because the environmental cues no longer predicted the platform position. We tried to circumvent this problem in Experiment 2 by testing the rats in a new environment. In the present study, two different procedures were used to evaluate the use of spatial cues by the young and the old rats. Because it appeared that the old rats did not use spatial cues in either procedure, we assume that the results cannot be explained in terms of rigidity, proactive interference, or response flexibility.

It could be argued that the old rats did not show a spatial bias in Experiment 2 because they were given insufficient training (only 28 trials). However, there was no indication that the old Lewis rats showed a spatial bias toward the training quadrant after 28 trials in Experiment 2 (see Figure 4). In several other studies with old Lewis rats (see also Blokland, Prickaerts, \& Raaijmakers, 1994; Blokland \& Raaijmakers, 1993), we found that the asymptotic performance always converges on 25-30 sec. This level of performance corresponds with the performance of the old rats in Experiment 2. We therefore assume that the lack of a spatial bias in the old Lewis rats was not caused by insufficient training.

Our data clearly show that the old Lewis rats did not use an allocentric strategy in the Morris task. Pelleymounter et al. (1987) evaluated the performance of old rats in the Morris task by using a procedure in which a salient configuration of stimuli was available to them. It was assumed that the simplicity and salience of these stimuli would approximate the features of a visual discrimination task - a type of task for which no agerelated deficits have been reported (e.g., Sarter \& Markowitsch, 1983). They found that the performance of the old rats was still impaired, and they concluded 
that age-related differences in performance in the Morris task cannot be attributed to perceptual deficits, but to an age-related impairment in the performance of a complex cognitive operation. This finding, however, could also be interpreted as evidence that old rats do not use distal spatial cues in this task. This latter interpretation would be in agreement with the findings of our study.

Procedural differences between the Morris and conefield tasks could have led to the different outcomes of the Morris and cone-field task experiments. For example, exposure to the test environment was longer in the cone-field task (trials of 7.5 min, maximum) than in the Morris task, and the rats were given more trials in the former task than in the latter ( 60 and 44 , respectively). Differences in exposure time could therefore explain the differences between the results of the cone-field and Morris maze studies. Taken together, there are at least three factors that could explain the age-related differences in performance in the cone-field and the Morris task: the aversiveness of the task, the motivation to perform the task, and the length of exposure to the task.

In addition, Lindner and Gribkoff (1991) reported that warming of old rats between trials in the Morris task improved spatial discrimination performance, suggesting that a loss of thermoregulatory control could confound the measures of cognitive function. Another possibility for the age-related performance deficit in the Morris task is that the physical condition of old rats is poor. We recently carried out an experiment in which we trained old Lewis rats extensively on a treadmill before they were used in the Morris task (unpublished data). The results indicated that, at the start of Morris task training, the performance of the pretrained rats was better than the performance of the nontrained rats. Apparently, the spatial discrimination performance of old rats is dependent on at least two factors in the Morris task - thermoregulation and physical condition-for which young and old rats differ.

In the present study we evaluated only the latency measures to analyze age-related differences in performance. It has been suggested that the latency measure may not be valid for comparing differences between rats of different ages because of age differences in motor performance (van der Staay \& de Jonge, 1993). However, the difference in escape latency between the young and the old rats was approximately $20 \mathrm{sec}$ in both Experiments 1 and 2. Furthermore, we found that all the old rats sometimes reached the platform within $10 \mathrm{sec}$, which suggests that age differences in motor perfomance cannot be the sole factor for the age-related difference in escape latency. Moreover, in two different procedures using measures that were independent of agerelated differences in motor performance, we showed that the old rats did not use spatial cues.

Finally, it could be argued that the age-related differences in performance in the Morris task could be caused by the duration of time that the rats were placed on the platform in the present study-3 sec. In most studies, rats are left on the platform for $60 \mathrm{sec}$, allowing them time to explore the environment. Recently, it was suggested that rats learn the location of the platform en route to the platform (Devan, Blank, \& Petri, 1992; Sutherland, Chew, Baker, \& Linggard, 1987). Apparently, performance is not improved when rats are placed on the platform for longer periods of time. In addition, we think that shorter times between the rats' finding of the platform and their removal from the platform increase the associative strength between swimming to the platform and escaping from the water (e.g., Dickinson, 1980).

The difference in the use of spatial cues by old Lewis rats in the different tasks suggests that the use of spatial information is independent of the ability to use spatial information. Age-related differences in spatial discrimination could therefore be caused by the inability to process spatial information, or because the task characteristics are such that the rats do not (efficiently) use spatial cues. For example, old rats do not use spatial cues in the Morris task, and young rats may develop a habit of inspecting nonreinforced places in a cone-field task. Consequently, before interpreting age-related differences in spatial discrimination performance in terms of age-related deficits in spatial information processing, first it has to be established whether both young and old rats can use spatial information to solve a task. In addition, it should be ruled out that the task characteristics affect the use of spatial information necessary to perform the task. We therefore suggest that agerelated differences in learning and memory should preferably be evaluated in different tasks, which are assumed to measure one cognitive dimension in one rat strain. This may lead to a better understanding of the nature of the age-related decline in learning and memory performance.

In conclusion, the present study showed that old Lewis rats do not use an allocentric orientation strategy in the spatial Morris task. The findings do not corroborate the results of earlier studies with old Lewis rats, showing that old rats use an allocentric orientation strategy (Blokland et al., 1992; van der Staay, Blokland, \& Raaijmakers, 1990). This discrepancy may be due to different operationalizations of place learning and the difference in the aversiveness of both tasks. The present results suggest that the use of a spatial orientation strategy is task dependent in both young and old Lewis rats. If old Lewis rats do not use an allocentric orientation strategy in the Morris task, but do use spatial cues in the cone-field task, age-related deficits in performance in the Morris task cannot be interpreted in terms of an impairment of spatial discrimination learning.

\section{REFERENCES}

AitKen, D. H., \& Meaney, M. J. (1989). Temporally graded, agerelated impairments in spatial memory in the rat. Neurobiology of Aging, 10, 273-276.

Barnes, C. A., NADEL, L., \& Honig, W. K. (1980). Spatial memory deficit in senescent rats. Canadian Journal of Psychology, 34, 29-39. 
Blokland, A., Prickaerts, J., \& Raaijmakers, W. (1994). Absence of impairments in spatial and temporal discrimination learning in Lewis rats after chronic ethanol consumption. Pharmacology, Biochemistry \& Behavior, 46, 27-34.

Blokland, A., \& RaAismakers, W. (1993). Correlation between behavioral and biochemical parameters in young and old Lewis rats. Behavioral \& Neural Biology, 60, 52-61.

Blokland, A., RaAimakers, W., Honig, W., \& van der Staay, F. J. (1992). Spatial discrimination learning and orientation strategy in young and old Lewis rats. Neuroscience Research Communications, 10, 105-110.

Devan, B. D., Blank, G. S., \& Petri, H. L. (1992). Place navigation in the Morris water task: Effects of reduced platform interval lighting and pseudorandom platform positioning. Psychobiology, 20, 120-126.

DiCkinson, A. (1980). Contemporary animal learning theory. Cambridge: Cambridge University Press.

DunN, O. J. (1961). Multiple comparisons among means. Journal of the American Statistical Association, 56, 52-64.

Dunnett, S. B., Evenden, J. L., \& Iversen, S. D. (1988). Delaydependent short-term memory deficits in aged rats. Psychopharmacology, 96, 174-180.

Dunnett, S. B., Martel, F. L., \& Iversen, S. D. (1990). Proactive interference effects on short-term memory in rats: II. Effects in young and aged rats. Behavioral Neuroscience, 104, 666-670.

GAGE, F. H., DunNetT, S. B., \& BJörkiund, A. (1984). Spatial learning and motor deficits in aged rats. Neurobiology of Aging, 5, 4348.

Gallagher, M., \& Pelleymounter, A. (1988). Spatial learning deficits in old rats: A model for memory decline in the aged. Neurobiology of Aging, 9, 549-556.

Goodrick, C. L. (1975). Behavioral rigidity as a mechanism for facilitation of problem solving for aged rats. Journal of Gerontology, 30, 181-184.

INGRAM, D. K. (1985). Analysis of age-related impairments in learning and memory in rodent models. Annals of the New York Academy of Sciences, 444, 312-331.

LINDNER, M. D., \& GRIBKoFF, V. K. (1991). Relationship between performance in the Morris water task, visual acuity, and thermoregulatory function in aged F344 rats. Behavioural Brain Research, 45, 45-55.

Lindner, M. D., \& Schallert, T. (1988). Aging and atropine effects on spatial navigation in the Morris water task. Behavioral Neuroscience, 102, 621-634.

Markowska, A. L., Stone, W. S., Ingram, D. K., Reynolds, J., Gold, P. E., Conti, L. H., Pontecorvo, M. J., Wenk, G. L., \& OLton, D. S. (1989). Individual differences in aging: Behavioral and neurobiological correlates. Neurobiology of Aging, 10, 31-43.

MoRrIs, R. G. M. (1981). Spatial localization does not require the presence of local cues. Learning \& Motivation, 12, 239-261.

Morris, R. G. M., Garrud, P., Rawlins, J. N. P., \& O'Keefe, J. A. (1982). Place navigation impaired in rats with hippocampal lesions. Nature, 297, 681-683.

Morris, R. G. M., SchenK, F., Tweedie, F., \& JarRard, L. E. (1990). Ibotenate lesions of hippocampus and/or subiculum: Dissociating components of allocentric spatial learning. European Journal of Neuroscience, 2, 1016-1028.
Olton, D. S. (1988). Aging and spatial cognition. Neurobiology of Aging, 9, 569-570.

Pelleymounter, M. A., Smith, M. Y., \& Gallagher, M. (1987). Spatial learning impairments in aged rats trained with a salient configuration of stimuli. Psychobiology, 5, 248-254.

Raaijmakers, W., Blokland, A., \& van der Staay, F. J. (1993). Spatial discrimination learning in rats as an animal model of $\operatorname{cog}$ nitive ageing. Behavioural Processes, 30, 165-174.

Rapp, P. R., Rosenberg, R. A., \& Gallagher, M. (1987). An evaluation of spatial information processing in aged rats. Behavioral Neuroscience, 101, 3-12.

RigTER, H. (1971). Pitfalls in behavioural ageing research in animals. In W. H. Gispen \& J. Traber (Eds.), Aging of the brain (pp. 197208). Amsterdam: Elsevier.

SARTER, M. (1987). Measurement of cognitive abilities in senescent animals. International Journal of Neuroscience, 32, 765-774.

SARTER, M., \& MARKowitsch, H. J. (1983). Reduced resistance to progressive extinction in senescent rats: A neuroanatomical and behavioral study. Neurobiology of Aging, 4, 203-215.

Stephens, D. N., Weidmann, R., Quartermain, D., \& Sarter, M. (1985). Reversal learning in senescent rats. Behavioural Brain Research, 17, 193-202.

Sutherland, R. J., Chew, G. L., Baker, J. C., \& Linggard, R. C. (1987). Some limitations on the use of distal cues in place navigation by rats. Psychobiology, 15, 48-57.

van der StaAy, F. J., Blokland, A., \& RaAijmakers, W. (1990). Different time course for age-related changes of behavior in a complex spatial cone-field discrimination task in Lewis rats. Psychobiology, 18, 305-311.

VAN DER STAay, F. J., \& DE JoNGE, M. (1993). Effects of age on water escape behavior and on repeated acquisition in rats. Behavioral \& Neural Biology, 60, 33-41.

van der Staay, F. J., Krechting, B., Blokland, A., \& RaalJMAKERS, W. G. M. (1990). The cone-field: A spatial discrimination task for the automatic and simultaneous assessment of working and reference memory in rats. Journal of Neuroscience Methods, 31 13-22.

van LuiJtelaar, E. L. J. M., van der StaAy, F. J., \& Kerbusch, J. M. L. (1989). Spatial memory in rats: A cross validation study. Quarterly Journal of Experimental Psychology, 41 B, 287-306.

Wallace, J. E., Krauter, E. E., \& Campbell, B. A. (1980). Animal models of declining memory in the aged: Short-term memory and spatial memory in the aged rat. Journal of Gerontology, 35, 355363.

Whishaw, I. Q. (1985). Cholinergic receptor blockade in the rat impairs locale but not taxon strategies for place navigation in a swimming pool. Behavioral Neuroscience, 99, 979-1005.

WhisHAW, I. Q. (1987). Hippocampal, granule cell and CA3-4 lesions impair formation of a place learning-set in the rat and induce reflex epilepsy. Behavioural Brain Research, 24, 59-72.

WINER, B. J. (1971). Statistical principles in experimental designs (2nd ed.). New York: McGraw-Hill.

(Manuscript received June 29, 1993 revision accepted for publication November 29, 1993.) 